\title{
Loss of autism-linked gene alters synapse development in mice
}

\author{
BY LAURA DATTARO
}

25 JANUARY 2022

\section{Listen to this story:}

https://www.spectrumnews.org/wpcontent/uploads/2022/01/audio-31852f61-ee8f-4361-a06d-7f18d419fa86-encodings.mp3

Loss of the autism-linked gene ASH1L leads to excess synapses and autism-like behaviors in mice, according to a study published today in Neuron. Boosting the activity of another gene that is downregulated in the mice as a result of the loss of $\mathrm{ASH} 1 \mathrm{~L}$ reverses some of those changes, the researchers also found.

Some research suggests that autistic people have altered synaptic pruning - the brain's process of editing unnecessary neuronal connections in early childhood. The fact that targeting an ASH1Lregulated gene restored this process provides a "promising and more specific therapeutic approach" for autistic people with ASH1L mutations, says Jin He, assistant professor of biochemistry at Michigan State University in East Lansing, who was not involved in the study.

The new work, led by Ji-Song Guan, associate professor of neuroscience at ShanghaiTech University in China, is also the first to show that loss of just one copy of ASH1L can cause autismlike traits in mice, He adds. Last year, He and his colleagues created the first ASH1 L knockout mice missing both copies of the gene in their brains. But the new mice, missing just one copy, more closely model what happens in autistic people who have ASH1L mutations, He says. Guan did not respond to requests for comment.

The ASH1L mice appear to have trouble differentiating between sounds, a challenge sometimes seen in people with autism. Connecting an autism gene with synapse formation and auditory 


\section{Spectrum | Autism Research News}

https://www.spectrumnews.org

challenges is exciting, says Sofia Lizarraga, assistant professor of neurobiology at the University of South Carolina in Columbia, who was not involved in the work.

"It was very elegantly done," Lizarraga says. "Looking at that connection with the auditory discrimination and synapse elimination is actually pretty novel."

Guan and his colleagues ran the ASH1L mice through a battery of behavioral tests. Unlike control mice, the ASH1L mice did not spend more time with an unfamiliar mouse than a new one, suggesting a difference in social behavior. By comparison, they also made fewer vocalizations to their mothers as pups, showed more anxiety and spent more time burying marbles, an activity considered akin to repetitive behaviors in people with autism.

When the team trained the mice to lick a waterspout after hearing a specific tone, the ASH1L mice learned the task as well as control mice. But new tones more frequently prompted them to lick than control mice, suggesting they have difficulty discriminating between sounds.

Cortical neurons from ASH1L mice cultivated in a dish had more branching arms, called dendrites, than those from control mice. They also eliminated fewer connections than the neurons of controls did after the researchers activated the cells.

Similarly, ASH1L mice had fewer pruned synapses than controls did after learning to associate a specific sound with a mild electric shock. The findings suggest that ASH1L helps regulate activitydependent synaptic pruning, which would explain the abundance of synapses in mice missing a copy of $\mathrm{ASH} 1 \mathrm{~L}$, the investigators write in the paper.

Hundreds of genes showed altered expression in the ASH1L mice at both 1 month and 1 year of age. Many of the genes are involved in synapse development in the auditory cortex and dorsal striatum, a brain region that facilitates movement. One gene in particular, EPHA7, had low expression levels in both areas at both ages.

Stimulating EPHA7 using a molecule that binds to it in ASH1L neurons increased the cells' synaptic pruning. ASH1L mice trained on the water-lick task performed as well as control mice after receiving an injection of the treatment to the auditory cortex.

The findings identify new molecular and cellular mechanisms that might underlie some autism traits, He says, although it's unlikely that all of them are tied to EPHA7. Future work should examine how other genes regulated by ASH1L contribute to autism, he says.

The team could also investigate what happens when ASH1L is knocked out in cells other than neurons, such as microglia, Lizarraga says.

Cite this article: https://doi.org/10.53053/NDF02643 